\title{
ASSORTATIVE MATING AND INTELLIGENCE: AN ANALYSIS OF PEDIGREE DATA
}

\author{
L. J. EAVES \\ Department of Genetics, University of Birmingham, Birmingham B15 2TT
}

Received 1.v.72

\begin{abstract}
SUMMARY
The I.Q. scores of 3558 individuals from a pedigree study were subjected to a biometrical genetical analysis. Only models which took account of assortative mating provided a statistically adequate summary of the observed variation for intelligence. The conclusions of this study agreed with a published analysis of other data in demonstrating a large additive genetical component of variation and the effect of assortative mating. Although the study detected no significant dominance, it was shown on theoretical grounds that the observed data were consistent with complete dominance for I.Q.
\end{abstract}

\section{INTRODUGTION}

WHILST it is an easy matter to detect a marital correlation for human traits it is often more difficult to detect the genetical consequences of assortative mating. Fisher (1918) proposed a genetical model of continuous human variation which incorporated the effects of assortative mating. Fisher's model was employed by Burt and Howard (1956) in their analysis of intelligence and, more recently, by Jinks and Fulker (1970) when they applied the techniques of biometrical genetics to the analysis of Burt's correlations between relatives for I.Q. (Burt, 1966). Jinks and Fulker proposed a test for the genetical consequences of assortative mating based on a comparison of within- and between-family components of genetical variation, but showed that the test was ineffective in the presence of dominance. They were able to detect assortative mating in the presence of dominance by including the parent-offspring correlation in their analysis to provide an estimate of additive genetical variation. Although the effect of common environments is small for I.Q., Jinks and Fulker suggest that these effects may inflate the estimate of additive variation leading to an underestimation of dominance. The scaling test they propose, furthermore, requires comparing estimates of components of variance for which standard errors are large, and involves the observed value of the marital correlation as a further parameter in the specification of the model.

It is possible, in theory and in practice, to develop a scaling test for the detection of assortative mating which involves data on only one generation. The method to be discussed does not require an estimate of the marital correlation but can be used to predict the marital correlation as a desirable independent validation of the model. The solution is achieved by the direct fitting of genetical models to the meansquares derived by a hierarchical analysis of variance of the individuals in the last generation of manygeneration pedigrees. 


\section{ThE DATA}

I.Q. scores were analysed for 3558 individuals from a number of pedigrees of Reed and Reed's (1965) family study of mental retardation. Only pedigrees with fully documented relationships for at least five generations were selected. The number of individuals was chosen to be the smallest likely to discriminate between various models with reasonable confidence (Eaves, 1972). Within each pedigree some data were omitted to clarify the genetical interpretation of the statistical analysis:

1. Offspring of known consanguineous matings were excluded.

2. Where an individual married more than once the offspring of only one marriage were included.

3. Individuals appearing in more than one pedigree and their offspring, were excluded.

4. In the case of like-sexed twins one individual of the pair was omitted because the zygosity of the pairs was unknown.

Two further assumptions, applicable to any genetical analysis of Reed and Reed's data are necessitated by the structure of the original sample:

1. That the selection of pedigrees with at least one instance of mental retardation still provides a sufficiently representative sample of the population for a genetical analysis of continuous variation.

2. That any missing I.Q. records are randomly distributed throughout the sample.

The I.Q. scores given in the study are those achieved nearest the age of 14 years, and where scores on both group and individual tests were available the authors adopted the latter on account of their greater reliability. No overall mean and variance is quoted for the study, which involves data on over 80,000 individuals. A mean of $105.44(\mathcal{N}=10767)$ is obtained by combining the figures given for the means of 1866 male spouses, 1866 female spouses, 1047 children of normal persons with retarded siblings, and 5988 children of normal persons without retarded siblings. The variance of these data is 224.94 . The sample analysed in this paper has a mean of 105.06 and a variance of $197 \cdot 18$ which may indicate that the sample is a little underrepresentative of the total variation. It is difficult to assess the consequences of this difference for a genetical analysis. The number of individuals excluded from a subset of 1351 individuals was 102 , i.e. $7 \cdot 5$ per cent. of the total available scores were excluded for various reasons. The figure is perhaps uncomfortably large and may result in the genetical model which emerges from the analysis being unrealistic as a model for the variation in the reference population. In spite of these difficulties it was decided to proceed with the analysis because sufficient is known about the genetical system underlying differences in intelligence for any major discrepancies to be immediately apparent. Indeed, it will be shown that the results of this analysis are clearly consistent with the outcome of other research on the genetics of intelligence.

The data analysed here usually relate to the fifth or sixth generation descendants of the grandparents of individuals institutionalised for mental retardation in the period 1911-18. This means that the scores are fairly contemporaneous and may, therefore, not be so susceptible to differences in 
test procedure and standardisation as scores on ancestral generations. The relative contemporaneity of scores on collateral relatives is a further advantage of the analysis to be presented in comparison with the analysis of data on successive generations.

\section{ANALysis of VARIANGe AND COMPONENTS OF VARIATION}

In addition to actual I.Q. scores on 3558 individuals, Reed and Reed's data allow the complete specification of the pedigree relationships of every individual in this study as far back as the great-great-grandparents. The descendants of 53 pairs of great-great-grandparents are analysed here. Within each great-great-grandparent the contribution of each greatgrandparent may be specified, and within each great-grandparent we may define the effect of each grandparent. This hierarchical model can thus be followed until finally the score of each individual within a family can be specified in terms of the contribution of his successively more remote ancestors.

The total variance, $\sigma_{T}^{2}$, may thus be partitioned into its components.

$$
\sigma_{T}^{2}=\sigma_{b g g g p}^{2}+\sigma_{g g p}^{2}+\sigma_{g p}^{2}+\sigma_{p}^{2}+\sigma_{w}^{2}
$$

where: $\sigma_{b g g g p}^{2}=$ between great-great-grandparents component of variance;

$\sigma_{g g p}^{2}=$ within great-great-grandparents, between great-grandparents component;

$\sigma_{g p}^{2}=$ within great-grandparents, between grandparents component;

$\sigma_{p}^{2}=$ within grandparents, between parents component;

$\sigma_{w}^{2}=$ within families component of variance.

The analysis of variance is presented in table 1 . No direct $F$ test of the variance components is possible because the highly unbalanced structure of

TABLE 1

Analysis of variance

\section{Item}

Between great-great-grandparents (GGGP)

Within great-great-grandparents: between great grandparents (GGP)

Within great grandparents:

between grandparents (GP)

Within grandparents:

between parents $(\mathrm{P})$

Within families (W)

$\begin{array}{rc}\text { d.f. } & \text { M.S. } \\ 52 & 971.8298 \\ 113 & 554.5149 \\ & \\ 401 & 308.5967 \\ & \\ 902 & 234 \cdot 3704 \\ 2089 & 121.6629\end{array}$

the hierarchy results in the coefficients of the same variance component changing from one meansquare to another. The expectations of mean squares and the estimates of the variance components are given in table 2. The coefficients of the $\sigma^{2}$ 's are calculated by an extension of the method outlined by Snedecor (1956). The standard errors of the components, which enable their significance to be tested, are obtained as the square roots of the diagonal elements of the inverse of the information matrix.

$$
\mathbf{M}=\left(\mathbf{C}^{\prime} \mathbf{W C}\right)^{-1}
$$

where $\mathbf{C}$ is the matrix of the coefficients of the variance components in the 
expectations of meansquares, and $\mathbf{W}$ is the diagonal matrix of weights. The elements of $\mathbf{W}$ are the reciprocals of the theoretical variances of the observed meansquares and are thus obtained from

$$
W_{i}=d_{i} / 2 x_{i}^{2}
$$

where $W_{i}$ is the $i$ th diagonal element of the matrix $\mathbf{W}$,

$d_{i}$ is the number of d.f. corresponding to the observed variance $x_{i}$.

From table 2 it can be seen that all the components of variance are significant beyond the 5 per cent. level with the exception of $\sigma_{b g g g p}^{2}$ which would be significant only at the 13 per cent. level. Four components possibly five, are thus required by the statistical model to account for the observed variation in I.Q. Presumably, therefore, this would form the necessary minimum for any interpretation of I.Q. differences based solely on chance and cultural factors. It will be apparent, however, from the analysis to follow that a greater economy can be achieved by relating the components

TABLE 2

Expectations of meansquares and estimates of variance components

\begin{tabular}{|c|c|c|c|}
\hline Item & E.M.S. & $\hat{\sigma}^{2}$ & S.E. \\
\hline GGGP & $\sigma_{w}^{2}+2.9496 \sigma_{p}^{2}+9 \cdot 0202 \sigma_{g p}^{2}+25 \cdot 7406 \sigma_{g g p}^{2}+66.6369 \sigma_{g g g p}^{2}$ & $5 \cdot 1269$ & $4 \cdot 54$ \\
\hline GGP & $\sigma_{w}^{2}+2 \cdot 9548 \sigma_{p}^{2}+7 \cdot 8901 \sigma_{g p}^{2}+19 \cdot 3830 \sigma_{g g p}^{2}$ & $9 \cdot 5719 *$ & 5.85 \\
\hline GP & $\sigma_{w}^{2}+2 \cdot 3754 \sigma_{p}^{2}+5 \cdot 4514 \sigma_{g p}^{2}$ & $13 \cdot 3355^{*}$ & 6.34 \\
\hline $\mathbf{P}$ & $\sigma_{w}^{2}+2 \cdot 3436 \sigma_{p}^{2}$ & $48 \cdot 0916 * * *$ & $7 \cdot 02$ \\
\hline W & $\sigma_{w}^{2}$ & $121 \cdot 6629 * * *$ & $5 \cdot 32$ \\
\hline
\end{tabular}

of variance, and consequently the observed meansquares, to their expectations on the basis of a genetical model.

\section{The genetical MODEL}

Fisher (1918) pioneered the genetical interpretation of the correlations between relatives. His work has been the subject of a detailed commentary by Moran and Smith (1966), has been summarised in relation to intelligence by Burt and Howard (1956) and his particular treatment of assortative mating has been outlined by Crow and Felsenstein, 1968). Fisher's model forms the basis of the subsequent analysis and it will be sufficient here to relate the expectations which Fisher gives for correlations to the expectations of the meansquares obtained in this particular analysis.

For a population in equilibrium under assortative mating the correlation between pairs of collateral relatives having one pair of common ancestors is given by

$$
r_{n}=c_{1} c_{2}\left(\frac{1+A}{2}\right)^{n}+\frac{1}{2}^{(n+1)} A^{(n-1)} c_{1}\left(1-c_{2}\right) .
$$

The parameters are defined as follows:

$A$ is the correlation between the additive genetical deviations of spouses; $c_{1}$ is the proportion of the total variation which can be ascribed to genetical differences (i.e. the "broad heritability"); 
$c_{2}$ is the proportion of the genetical variation which is additive;

$n$ is the number of opportunities for genetical recombination in the shortest path in the pedigree linking the relatives under consideration (i.e. $n=1$ for sibs, 3 for first cousins).

The additive variation which comprises the numerator of $c_{2}$ is made up of that part which would result from random mating a population with the same gene frequencies and an additional fraction which arises because of assortative mating.

It can be shown quite simply that $c_{1} c_{2}$ is the proportion of the total variation which is due to additive genetical effects (i.e. the " narrow heritability", $\left.h t_{n}\right)$ and $c_{1}\left(1-c_{2}\right)$ is the proportion of the total variation which is due to dominance effects.

Since the correlation $r_{n}$ is the ratio of the variance between groups of $n$th degree relatives, $\sigma_{b n}^{2}$ to the total variance, $\sigma_{T}^{2}$

$$
\text { i.e. } r_{n}=\sigma_{b n}^{2} / \sigma_{T}^{2}
$$

we may write the expectation of $\sigma_{b n}^{2}$ as

$$
\sigma_{b n}^{2}=r_{n} \sigma_{T}^{2}
$$

Or, in terms of Fisher's model

$$
\sigma_{b n}^{2}=\sigma_{T}^{2}\left[c_{1} c_{2}\left(\frac{1+A}{2}\right)^{n}\right]+\sigma_{T}^{2}\left[\frac{1}{2}{ }^{(n+1)} A^{(n-1)} c_{1}\left(1-c_{2}\right)\right]
$$

Now $\sigma_{T}^{2}$ cancels in both parts of the right-hand side of this expression since $c_{1} c_{2}$ and $c_{1}\left(1-c_{2}\right)$ are both ratios involving $\sigma_{T}^{2}$ as denominator. This leaves, again in Fisher's notation:

$$
\begin{aligned}
\sigma_{b n}^{2} & =\left(\tau^{2}+\frac{A}{1-A} \tau^{2}\right)\left(\frac{1+A}{2}\right)^{n}+\frac{1}{2}^{(n+1)} A^{(n-1)} \epsilon^{2} \\
& =\tau^{2}\left(\frac{1}{1-A}\right)\left(\frac{1+A}{2}\right)^{n}+\frac{1}{2}^{(n+1)} A^{(n-1)} \epsilon^{2}
\end{aligned}
$$

where $\tau^{2}$ is the additive genetical component of variation, and

$\epsilon^{2}$ is the dominance genetical component.

It is convenient at this stage to relate $\tau^{2}$ and $\epsilon^{2}$ to the more familiar notation of biometrical genetics (Mather and Jinks, 1971) and write finally,

$$
\begin{aligned}
\tau^{2} & =\frac{1}{2} D_{R} \\
\epsilon^{2} & =\frac{1}{4} H_{R}
\end{aligned}
$$
sibs,

When $n=1$, that is for the variance component between families of full

$$
\sigma_{b p}^{2}=\frac{1}{2} D_{R}\left(\frac{1}{1-A}\right)\left(\frac{1+A}{2}\right)+\frac{1}{16} H_{R}
$$

which reduces to the expectation given by Jinks and Fulker (1970) namely:

$$
\begin{aligned}
\sigma_{b p}^{2} & =\frac{1}{2} D_{R}\left(\frac{1+A}{2(1-A)}\right)+\frac{1}{16} H_{R} \\
& =\frac{1}{4} D_{R}+\frac{1}{2}\left(\frac{A}{1-A}\right) D_{R}+\frac{1}{16} H_{R} .
\end{aligned}
$$


The expectations of $\sigma^{2}$ for the other degrees of collateral relatives are given in table 3. Additional parameters may be added to the model to specify environmental effects should these be required.

TABLE 3

Expectations of components of variance

\begin{tabular}{llcll}
$\sigma^{2}$ & $n$ & $D_{R}$ & \multicolumn{2}{c}{$H_{R}$} \\
$\sigma_{b g g g p}^{2}$ & 7 & $\frac{1}{2}\left(\frac{1}{1-A}\right)\left(\frac{1+A}{2}\right)^{7}$ & $\frac{1}{2}^{10}$ & $A^{6}$ \\
$\sigma_{b g g p}^{2}$ & 5 & $\frac{1}{2}\left(\frac{1}{1-A}\right)\left(\frac{1+A}{2}\right)^{5}$ & $\frac{1}{2}^{8}$ & $A^{4}$ \\
$\sigma_{b g p}^{2}$ & 3 & $\frac{1}{2}\left(\frac{1}{1-A}\right)\left(\frac{1+A}{2}\right)^{3}$ & $\frac{1}{2}^{6}$ & $A^{2}$ \\
$\sigma_{b p}^{2}$ & 1 & $\frac{1}{2}\left(\frac{1}{1-A}\right)\left(\frac{1+A}{2}\right)$ & $\frac{14}{2}$ & \\
$\sigma_{w}^{2}$ & - & $\frac{1}{4}$ & $\frac{3}{16}$
\end{tabular}

It is not possible to relate the $\sigma^{2}$ 's in the table directly to those in the hierarchical analysis except in the case of $\sigma_{w}^{2}$ and $\sigma_{b g g g p}^{2}$. The $\sigma^{2}$ 's at the intermediate levels have to be adjusted to take account of the variation removed by components at higher levels in the hierarchy.

Thus,

$$
\begin{aligned}
\sigma_{g g p}^{2} & =\sigma_{b g g p}^{2}-\sigma_{b g g g p}^{2} \\
\sigma_{g p}^{2} & =\sigma_{b g p}^{2}-\sigma_{b g g p}^{2} \\
\sigma_{p}^{2} & =\sigma_{b p}^{2}-\sigma_{b g p}^{2} .
\end{aligned}
$$

At this stage the coefficients of the expectations are better evaluated numerically than algebraically using the algebraic coefficients of table 3 and substituting for particular values of $A$. A column vector of coefficients, $\mathbf{b}_{i}$, is thus generated for the $i$ th parameter of the model. Multiplying the original matrix of the coefficients of the variance components, $\mathbf{C}$, by each $\mathbf{b}_{i}$ gives the coefficients of the parameters of the genetical model in the expectations of the observed statistics,

$$
\text { i.e. } \mathbf{z}_{i}=\mathbf{A} \mathbf{b}_{i} \text {. }
$$

The individual vectors, $\mathbf{z}_{i}$, may be combined into a matrix $\mathbf{Z}$ which summarises the coefficients of all the genetical parameters in the expectations of meansquares of the hierarchical analysis.

\section{Fitting the Genetical MOdeL}

Providing the number of parameters, $p$, is less than the number of observed statistics, $k$, we may obtain weighted least-squares estimates of the parameters by solving the equations

$$
\left(\mathbf{Z}^{\prime} \mathbf{W Z}\right)^{-\mathbf{1}}\left(\mathbf{Z}^{\prime} \mathbf{W} \mathbf{x}\right)=\hat{\boldsymbol{\theta}}
$$

where $\mathbf{Z}$ and $\mathbf{W}$ are the matrices of coefficients and weights as already defined, $\mathbf{x}$ the vector of observed statistics, and $\hat{\theta}$ is the vector of estimates. The application of this procedure to genetical analysis is discussed by Cooke et al. (1962). The sum of the weighted squared deviations of the observed statistics from their expectations when the model is fitted is a chi-square for $(k-p)$ d.f., and can be used to test the goodness of fit of the model. By 
deleting redundant parameters, or fitting additional parameters when necessary it is possible to arrive at the simplest model which will account for the observed variation (Jinks, Perkins and Gregory, 1972).

The basic genetical model fitted to the data included four parameters: $D_{R}$ and $H_{R}$ specifying additive and dominance genetical variation, and $E_{1}$ and $E_{2}$ specifying within-family and between-family environmental influences. With no independent estimate of $E_{2}$ from groups of separated twins or sibs it is not possible to separate $H_{R}, E_{1}$ and $E_{2}$ with the result that the information matrix is singular. Only three independent parameters can be fitted. Either $E_{2}$ can be estimated on the assumption that $H_{R}$ is zero, or $H_{R}$ can be estimated on the assumption that $E_{2}$ is zero. Since the full model involving four parameters gives an information matrix of rank 3 the additional parameter is redundant with this set of data and both three-parameter models will give the same chi-square test of the residual variation.

The genetical model was fitted firstly on the assumption of random mating and subsequently assuming various degrees of assortative mating.

\section{Testing the assumption of RANDOM Mating}

Table 4 gives the expectations of meansquares in terms of the genetical and environmental parameters on the assumption that $A=0$. Fitting

TABLE 4

Expectations of meansquares for genetical model

\begin{tabular}{lcccc} 
& \multicolumn{4}{c}{ 1. Random mating } \\
\cline { 2 - 5 } Meansquare & $D_{R}$ & $H_{R}$ & $E_{1}$ & $E_{\mathbf{2}}$ \\
GGGP & 1.7878 & 0.3719 & 1 & 2.9496 \\
GGP & 1.4010 & 0.3722 & 1 & 2.9548 \\
GP & 0.9509 & 0.3360 & 1 & $2 \cdot 3754$ \\
P & 0.6894 & 0.3340 & 1 & $2 \cdot 3436$ \\
W & 0.2500 & 0.1875 & 1 & 0
\end{tabular}

various combinations of the parameters gives the estimates and significance levels presented in table 5. No two-parameter model fits the data if we

TABLE 5

Estimates of genetical and environmental parameters (random mating model)

\begin{tabular}{|c|c|c|c|c|c|c|c|}
\hline Model & $D_{R}$ & $H_{R}$ & $E_{1}$ & $E_{2}$ & $x^{2}$ & d.f. & $\mathrm{P}$ \\
\hline 1 & $401 \cdot 44 * * *$ & - & - & - & $59 \cdot 82$ & 4 & $* * *$ \\
\hline 2 & $187.98 * * *$ & $380.35 * * *$ & - & - & $15 \cdot 70$ & 3 & * \\
\hline 3 & $296 \cdot 07 * * *$ & - & $46 \cdot 31 * * *$ & - & $17 \cdot 31$ & 3 & \\
\hline 4 & $406 \cdot 01 * * *$ & $-476 \cdot 45^{*}$ & $109 \cdot 44 * * *$ & - & 4.89 & 2 & \\
\hline 5 & $405 \cdot 54 * * *$ & - & $20 \cdot 22 * * *$ & $-29 \cdot 68^{*}$ & 4.89 & 2 & \\
\hline
\end{tabular}

Dashes indicate that the particular parameters were deleted from the model.

assume random mating since all the chi-squares are significant beyond the 1 per cent. level. It is noticeable that the three parameter models, 4 and 5, give significant and negative estimates of $H_{R}$ and $E_{2}$ respectively. This result suggests a fundamental failure of the random mating model for I.Q 
One possible explanation, of course, may be the cultural determination if I.Q. differences, but a further, more tangible, possibility may be the effect of the mating system on the pattern of genetical variation.

\section{The EFFEct OF ASSORTAtive MAting}

The possible influence of assortative mating may be investigated by modifying the coefficients of the model to take account of some degree of assortative mating. Table 6 gives the expectations of meansquares for a

TABLE 6

Expectations of meansquares for genetical model

2. Assortative mating $A=0.3$

Meansquare
GGGP
GGP
GP
P
W

$\begin{array}{ccc}D_{R} & H_{R} & E_{1} \\ 5 \cdot 6281 & 0.3809 & 1 \\ 2.8638 & 0.3795 & 1 \\ 1.5045 & 0.3401 & 1 \\ 0.8791 & 0.3307 & 1 \\ 0.2500 & 0.1875 & 1\end{array}$

three parameter model when $A=0 \cdot 3$. The value of $A$ is chosen to be fairly consistent with the observed phenotypic correlation between mates for I.Q., but any similar value would suffice. Comparison of the model in table 6 with the random mating model in table 4 reveals how the contribution of the additive component, $D_{R}$, is greatly increased at the higher levels of the hierarchy when assortative mating is incorporated into the model.

Various combinations of parameters may be fitted as before, to give the results summarised in table 7. A simple additive genetical model, incorpora-

TABLE 7

Estimates of genetical and environmental parameters (assortative mating model $A=0.3$ )

$\begin{array}{ccccccc}\text { Model } & D_{R} & H_{R} & E_{1} & \chi^{2} & \text { d.f. } & \text { P } \\ 1 & 293 \cdot 75^{* * *} & - & - & 234.07 & 4 & * * * \\ 2 & 102 \cdot 41 * * * & 495 \cdot 51 * * * & - & 6.94 & 3 & \text { n.s. } \\ 3 & 163 \cdot 13 * * * & - & 81 \cdot 46 * * * & 1.51 & 3 & \text { n.s. } \\ 4 & 142.95 * * * & 141.09 & 59 \cdot 46 * * * & 0.74 & 2 & \text { n.s. } \\ * * * & =\text { Significant at } 0.001 \text { level. } \\ \text { n.s. } & =\text { Not significant at } 0.05 \text { level. }\end{array}$

ting no dominance variation or environmental variation, once more fails to fit the data. The additive-dominance genetical model almost fails if it is assumed that there is no environmental variation. The most striking difference between this table and the corresponding table for the random-mating model is the unequivocal fit of the two parameter model involving simply additive genetical variation and within family environmental variation $\left(\chi_{(3)}^{2}=1.51\right)$. The significance of this result is only emphasised by the failure of the $D_{R}, H_{R}$ model to provide any adequate description of the data with whatever mating system is assumed, and the failure of the $D_{R}, E_{1}$ model to account for the observed variation on the assumption of random mating. The method of analysis clearly permits the detection of the genetical consequences of assortative mating and in this particular instance reduces the number of necessary parameters from four to two, namely $D_{R}$ and $E_{1}$ 
Having thus decided on a minimal model it is possible to find the value of $A$ which minimises the chi-square in order to provide the best-fitting model for the observed statistics. Fitting models with other values of $A$ also provides an indication of the sensitivity of the analysis to changes in the degree of assortative mating assumed.

Table 8 gives the expectations of meansquares in terms of $E_{1}$ and $D_{R}$ for various values of $A$ from 0 (i.e. random mating) to $0 \cdot 8$. The very marked

TABLE 8

Reduced assortative mating models

\begin{tabular}{|c|c|c|c|c|c|c|c|c|}
\hline \multirow[b]{2}{*}{ Meansquare } & \multirow[b]{2}{*}{$E_{1}$} & \multicolumn{7}{|c|}{ Coefficient of $D_{R}$ for $A=$} \\
\hline & & $0 \cdot 0$ & $0 \cdot 1$ & $0 \cdot 2$ & $0 \cdot 3$ & $0 \cdot 4$ & $0 \cdot 6$ & 0.8 \\
\hline GGGP & 1 & $1 \cdot 7878$ & $2 \cdot 5260$ & $3 \cdot 7038$ & $5 \cdot 6269$ & $8 \cdot 8543$ & $24 \cdot 6540$ & 91.5345 \\
\hline GGP & 1 & $1 \cdot 40$ & 1.7 & $2 \cdot 2$ & 2.8 & 3.7 & 5 & $9 \cdot 6820$ \\
\hline GP & 1 & 0.9509 & $1 \cdot 1076$ & 1.2911 & $1 \cdot 5046$ & 1.7513 & $2 \cdot 3613$ & $3 \cdot 1530$ \\
\hline $\mathrm{P}$ & 1 & 0.6894 & 0.7494 & $0 \cdot 8125$ & $0 \cdot 8784$ & 0.9472 & 1.0938 & $1 \cdot 2520$ \\
\hline W & 1 & $0 \cdot 2500$ & 0.2500 & 0.2500 & 0.2500 & 0.2500 & 0.2500 & 0.2500 \\
\hline
\end{tabular}

increase of the coefficient of $D_{R}$ in the between great-great-grandparents meansquare as $A$ increases reflects the high degree of homozygosity and linkage disequilibrium when $A$ is large, and the comparatively small amount of segregation taking place.

Two additional models were also fitted in an attempt to minimise chisquare; for $A=0.25$, and $A=0.27$. The results of fitting the various models are summarised in table 9 . The sensitivity of the model to changes in $A$ is

TABle 9

\begin{tabular}{|c|c|c|c|c|c|}
\hline \multicolumn{6}{|c|}{ Summary of reduced models } \\
\hline$A$ & $\hat{D}_{R}$ & $\hat{E}_{1}$ & $\chi_{(\mathrm{s})}^{\mathbf{2}}$ & $h t_{n}$ & $\mu$ \\
\hline 0 & $296 \cdot 07 * * *$ & $46 \cdot 31 * * *$ & $17 \cdot 31 * * *$ & $0 \cdot 76$ & 0 \\
\hline $0 \cdot 1$ & $239 \cdot 23 * * *$ & $61 \cdot 01 * * *$ & $7 \cdot 43$ & 0.69 & $0 \cdot 145$ \\
\hline $0 \cdot 2$ & $199 \cdot 64 * * *$ & $71 \cdot 49 * * *$ & $2 \cdot 08$ & $0 \cdot 64$ & $0 \cdot 313$ \\
\hline $0 \cdot 25$ & $179 \cdot 81 * * *$ & $76 \cdot 88 * * *$ & $1 \cdot 26$ & $0 \cdot 61$ & 0.410 \\
\hline 0.27 & $173 \cdot 06 * * *$ & $78 \cdot 72 * * *$ & 1.25 & 0.60 & 0.450 \\
\hline $0 \cdot 3$ & $163 \cdot 13 * * *$ & $81 \cdot 46 * * *$ & $1 \cdot 51$ & 0.59 & 0.508 \\
\hline $0 \cdot 4$ & $134 \cdot 99 * * *$ & $89 \cdot 63 * * *$ & $5 \cdot 26$ & 0.56 & 0.714 \\
\hline 0.6 & $91 \cdot 86 * * *$ & $103 \cdot 17 * * *$ & $22 \cdot 27 * * *$ & 0.53 & $1 \cdot 132$ \\
\hline $0 \cdot 8$ & $61 \cdot 82 * * *$ & $114 \cdot 38 * * *$ & $47 \cdot 80 * * *$ & 0.57 & $1 \cdot 404$ \\
\hline
\end{tabular}

Note: All estimates, with the exception of those for $A=0 \cdot 08$, are true maximum likelihood estimates obtained by iteration.

obvious. The procedure adopted here can discriminate models involving little or no assortative mating and those incorporating a high degree of assortative mating from models which assume an intermediate value of $A$. Interpolation on a graph relating $\chi^{2}$ to $A$ suggested that values of $A$ between 0.09 and 0.43 would provide a satisfactory model and that a value of 0.27 approximates to that satisfying the minimum chi-square criterion. Values of $A$ outside the limits given would lead to failure of the model at or beyond the 5 per cent. level.

The proportion of the total variation which can be ascribed to additive genetical causes is:

$$
h t_{n}=\frac{1}{2(1-A)} D_{R} /\left[\frac{1}{2(1-A)} D_{R}+E_{1}\right] \text {. }
$$


The values obtained for $h t_{n}$ are given in table 9 . About 60 per cent. of the total variation is apparently due to additive genetical influences.

Since the value obtained for $A$ has been derived without any reference to the observed marital correlation the prediction of the latter from the parameters of the genetical model provides an important independent validation of the method. The additive genetical correlation between spouses is given by

$$
\begin{aligned}
A & =c_{1} c_{2} \mu, \text { in Fishers notation, } \\
& =h t_{n} \mu \text { in the present notation, }
\end{aligned}
$$

where $\mu$ is the marital correlation. Given $A$ and $h t_{n}$, we can obtain an estimate of $\mu$ which can be compared with the value observed in the population. The values of $\mu$ estimated by this method are given in table 9 . The estimates are clearly highly sensitive to changes in $A$ since $h t_{n}$ and $A$ are negatively correlated. When $A=0 \cdot 27, \mu=0.45$. Reed and Reed (1965) give 0.326 as the observed value for the marital correlation based on 1866 pairs, but when a correction for unreliability is applied their estimate of the "true" correlation is 0.464 which is remarkably close to the value obtained when $A=0 \cdot 27$. Either value for the observed correlation is well within the limits of this analysis since the lower limit for $A=0.09$ corresponds to $\mu=0.14$ and the upper limit of $A=0.43$ corresponds to $\mu=0.78$. Both of these figures are approximate, based on heritabilities derived by interpolation.

\section{Discussion}

This body of data, which forms part of perhaps the largest single study of I.Q., is clearly consistent with a simple genetical interpretation which takes account of the observed degree of assortative mating. The only other study which even approaches this magnitude is that by Burt (1966) and with which some comparison is clearly necessary. The biometrical-genetical reanalysis of these data by Jinks and Fulker (1970) provides the best basis for comparison.

In two important respects the two studies give directly comparable results, namely: the estimate of the narrow heritability and the estimate of $A$ which best satisfies the data. This analysis of Reed and Reed's data provides an estimate of $h t_{n}=0.60$ and $A=0.27$, Jinks and Fulker's analysis of Burt's correlations give $h t_{n}=0.66$ and $A=0 \cdot 26$. The similarity of these two results is sufficient vindication of the comparison. One striking dissimilarity between the two studies which cannot be dismissed lightly is the fact that no dominance was apparently detected in this analysis whereas Burt's data reveal significant dominance when the parent-offspring correlation is included in the analysis. Jinks and Fulker indicate that the estimate of $H_{R}$ they obtain is consistent with complete dominance for I.Q. if the gene frequencies are equal at all loci involved in the expression trait. Indeed, their own analysis of the within family skewness for Reed and Reed's data is indicative of directional dominance. It has been shown elsewhere (Eaves, 1972) that a study of the design and magnitude executed by Burt would be capable in many instances of detecting complete dominance. It may now be demonstrated using a similar procedure that the design and analysis employed in this paper is unlikely to yield a significant estimate of $H_{R}$ even if dominance were complete. 
Let the total variance of the population be unity. Let the narrow heritability be $0.60, A=0.3$ and let dominance be complete, i.e. $H_{R} / D_{R}=1 \cdot 0$. The theoretical value of $D_{R}$ and $H_{R}$ is then $D_{R}=H_{R}=0.86$. The broad heritability is thus 08.2 approximately, leaving 0.18 units of variation ascribed to environmental causes. Let all this variation be due to within family environmental differences, i.e. put $E_{1}=0 \cdot 18$. Following the procedure described by Eaves (1972), we may generate expected values for the meansquares of the hierarchical analysis and compute weights for the weighted least squares analysis for a sample of the size analysed in this study. The simulated data and their expectations are given in table 10. The information matrix and its inverse are given in table 11. Such data would yield estimates and standard errors as follows:

$$
\begin{aligned}
& D_{R}=0.86 \pm 0.14 \\
& H_{R}=0.86 \pm 0.88 \\
& E_{1}=0.18 \pm 0.14
\end{aligned}
$$

TABLE 10

The model and the expected statistics for a population of unit total variance when $A=0.3, D_{R}=H_{R}=0.86, E_{1}=0.18$

$\begin{array}{lccccr}\text { Meansquare } & D_{R} & H_{R} & E_{1} & x & \text { Weights } \\ \text { GGGP } & 5 \cdot 62 & 0.38 & 1 & 5 \cdot 34 & 0.91 \\ \text { GGP } & 2 \cdot 86 & 0.38 & 1 & 2.97 & 6.41 \\ \text { GP } & 1.50 & 0.34 & 1 & 1 \cdot 76 & 64.73 \\ \text { P } & 0.88 & 0.33 & 1 & 1 \cdot 22 & 303.01 \\ \text { W } & 0.25 & 0.19 & 1 & 0.56 & 3330.68\end{array}$

TABLE 11

The information matrix and the variance-covariance matrix of the parameters

$$
\begin{aligned}
& \begin{array}{lll}
D_{R} & H_{R} & E_{1}
\end{array}
\end{aligned}
$$

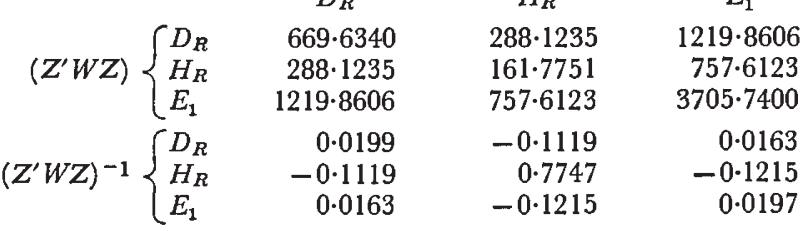

We can thus see that even with complete dominance the likelihood of its detection is small with a design of this type and we may therefore conclude that this analysis of Reed and Reed's data and Jinks and Fulker's analysis of Burt's data are consistent with complete dominance for I.Q. Having noted the likely consequences of fitting a dominance parameter to Reed and Reed's data we may consider the values obtained for $D_{R}, H_{R}$ and $E_{1}$ when these three parameters were fitted on the assumption that $A=0.3$ (table 7). A non-significant estimate of $H_{R}$ is obtained, which is precisely what is expected, but the estimate is at least consistent with complete dominance since it is almost equal to the estimate of $D_{R}$. The additional parameter, however, leads to no significant improvement in the fit of the model. Whatever dominance variation there is, has been accounted for by the estimation of $D_{R}$ and $E_{1}$ with which $H_{R}$ is highly correlated. The futility of attempting to fit a dominance parameter is indicated by the fact that 94 per cent. of the variation of the coefficients of $H_{R}$ in the model of table 10 may be accounted for by correlated variation in the coefficients of $D_{R}$ and $E_{1}$. 
Further evidence of the degree of independence of the parameters can be obtained from the eigenvalues of the information matrix (table 11) which are, in descending order: 4294.03, 241.89 and 1.23. These account for 94.64 per cent., 5.33 per cent. and 0.00 per cent. respectively of the total information. The fact that the last eigenvalue is small implies that there is a high degree of redundancy in the model and that the whole system could be adequately explained by reference to no more than two orthogonal parameters. It is, therefore, to be expected that the analysis presented here, whilst providing a comparatively sensitive test for the consequences of assortative mating offers a poor test of dominance.

In the light of this discussion it is clear that the data of Reed and Reed analysed here, and Burt's data give results which are highly similar with respect to the amount of additive genetical variation and the degree of assortative mating and provide results which are consistent with the assumption of complete dominance at the loci involved in the determination of individual differences in I.Q. The analysis presented in the paper has the particular advantage of detecting unambiguously the genetical consequences of assortative mating without reference to any estimate of the marital correlation except as a final stage in validation of the results by criteria external to the analysis.

Acknowledgment.-This work is part of a research programme in Psychogenetics supported by the British Medical Research Council. I am indebted to Professor J. L. Jinks for his suggestions and encouragement and to Dr D. W. Fulker for helpful discussion.

\section{REFERENCES}

BURT, c. 1966. The genetic determination of intelligence: a study of monozygotic twins reared apart. British Journal of Psychology, 57, 137-153.

BURT, C., AND HOWARD, M. 1956. The multifactorial theory of inheritance and its application to intelligence. British Fournal of Statistical Psychology, 9, 115-125.

COOKE, P., MORLEY-JONES, R., MATHER, K., BONSALL, G. W., AND NEDLER, J. A. 1962. Estimating components of continuous variation. I. Statistical. Heredity, 17, 115-133.

CROW, J. F., AND FELSENSTEIN, J. 1968. The effect of assortative mating on the genetic composition of a population. Eugenics Quarterly, 15, 85-97.

EAVES, L. J. 1972. Computer simulation of sample size and experimental design in human psychogenetics. Psychological Bulletin, 77, 144-152.

FISHER, R. A. 1918. The correlation between relatives on the supposition of Mendelian inheritance. Transactions of the Royal Society of Edinburgh, 52, 399-433.

JINKS, J. L., AND FULKER, D. W. 1970. A comparison of the biometrical-genetical, MAVA and the classical approaches to the analysis of human behaviour. Psychological Bulletin, $73,311-349$.

JINKS, J. L., PERKINS, J. M., AND GREGORY, R. s. 1972. The analysis and interpretation of differences between reciprocal crosses of Nicotiana rustica varieties. Heredity, 28, 363-377.

Mather, K., AND JiNks, J. L. 1971. Biometrical Genetics: the Study of Continuous Variation. Chapman and Hall, London.

MORAN, P. A. P., AND SMITH, с. A. B. 1966. Commentary on R. Fisher's Paper on the Correlation between Relatives on the Supposition of Mendelian Inheritance. Cambridge University Press, Cambridge.

REED, E. W., AND REED, s. c. 1965. Mental Retardation: a Family Study. W. B. Saunders, London.

SNEDECOR, G. W. 1956. Statistical Methods applied to Experiments in Agriculture and Biology (5th ed.). Ames: Iowa State Press. 